\title{
An asymptotically optimal gradient algorithm for quadratic optimization with low computational cost
}

\author{
Anatoly Zhigljavsky • Luc Pronzato · Elena Bukina
}

October 11, 2011

\begin{abstract}
We consider gradient algorithms for minimizing a quadratic function in $\mathbb{R}^{n}$ with large $n$. We suggest a particular sequence of step-lengthes and demonstrate that the resulting gradient algorithm has a convergence rate comparable with that of Conjugate Gradients and other methods based on the use of Krylov spaces. When the problem is large and sparse, the proposed algorithm can be more efficient than the Conjugate Gradient algorithm in terms of computational cost, as $k$ iterations of the proposed algorithm only require the computation of $O(\log k)$ inner products.
\end{abstract}

Keywords Quadratic optimization · gradient algorithms · conjugate gradient · arcsine distribution · Fibonacci numbers · estimation of leading eigenvalues

\section{Introduction}

Consider the problem of minimizing a quadratic function $f(\cdot)$ defined on $\mathbb{R}^{n}$ by

$$
f(x)=\frac{1}{2}(A x, x)-(x, b),
$$

where $(\cdot, \cdot)$ denotes the inner product. We assume that $A$ is a symmetric positive-definite matrix. Let $m=\lambda_{1} \leq \ldots \leq \lambda_{n}=M$ be the eigenvalues of $A$ (with $0<m<M<\infty$ ) and $\left\{q_{1}, \ldots, q_{n}\right\}$ be the set of corresponding orthonormal eigenvectors. We do not assume that any information is available about the eigenvalues $\lambda_{i}$ and eigenvectors $q_{i}, i=1, \ldots, n$ and suppose that the condition number $\rho=M / m$ may be large.

The work of E. Bukina was partially supported by the EU through a Marie-Curie Fellowship (EST-SIGNAL program: http://est-signal.i3s.unice.fr) under the contract Nb. MEST-CT-2005-021175. Part of this work was accomplished while the first two authors were invited at the Isaac Newton Institute for Mathematical Sciences, Cambridge, UK; the support of the INI and of CNRS is gratefully acknowledged.

A. Zhigljavsky

School of Mathematics, Cardiff University, Cardiff, CF24 4YH, UK. E-mail: ZhigljavskyAA@cardiff.ac.uk

L. Pronzato · E. Bukina

Laboratoire I3S, CNRS - UNS, 2000 route des Lucioles - B.P. 121, F-06903 Sophia Antipolis, France.

E-mail: pronzato@i3s.unice.fr E-mail: bukina@i3s.unice.fr 
Consider a general gradient algorithm with iterations of the form $x_{k+1}=x_{k}-\gamma_{k} g_{k},(k=$ $0,1, \ldots)$, where $x_{0} \in \mathbb{R}^{n}$ is a starting point, $\gamma_{k}>0$, the step-size at iteration $k$, is determined by some rule and $g_{k}=\nabla f\left(x_{k}\right)=A x_{k}-b$ is the gradient of the objective function $f(\cdot)$ at point $x_{k}$. These iterations can be rewritten in terms of the gradients as

$$
g_{k+1}=g_{k}-\gamma_{k} A g_{k}
$$

It is sometimes convenient to rewrite the basic iteration (2) as $g_{k}=P_{k}(A) g_{0}$, where $P_{k}$ denotes the polynomial $P_{k}(\lambda)=\left(I-\gamma_{0} \lambda\right)\left(I-\gamma_{1} \lambda\right) \ldots\left(I-\gamma_{k-1} \lambda\right)$.

We are interested in situations where the dimension $n$ can be very large; it could even be infinite, with $A$ a self-adjoint operator in a Hilbert space, but we shall restrict the presentation to the finite dimensional situation. We assume that $n$ is much larger than the number of iterations $k_{*}$ needed to achieve the precision required; formally, $k_{*}=o(n)$ with $k_{*} \rightarrow \infty$ in asymptotic considerations.

We shall define the rate of convergence at iteration $j$ by $r_{j}=\left(g_{j+1}, g_{j+1}\right) /\left(g_{j}, g_{j}\right)$. The rate of convergence of the algorithm (2) after $k$ iterations is then $R_{k}=\prod_{j=0}^{k-1} r_{j}=\left(g_{k}, g_{k}\right) /\left(g_{0}, g_{0}\right)$ and the asymptotic rate can naturally be defined as $R=\lim _{k \rightarrow \infty} R_{k}^{1 / k}$, if the limit exists. It is easy to see that $R$ does not depend on $g_{0}$ as long as $\alpha_{i} \neq 0$ for all $i$. Other rates of convergence, which are asymptotically equivalent to $r_{k}$, could be considered as well, see Th.6 in [7]. In particular, the rate $r_{k}^{\prime}=\left(f\left(x_{k+1}\right)-f\left(x^{*}\right)\right) /\left(f\left(x_{k}\right)-f\left(x^{*}\right)\right)$ is of great interest in optimization theory.

The method of Steepest-Descent (SD) chooses $\gamma_{k}$ in (2) that minimizes $r_{k}^{\prime}$; direct calculation gives $\gamma_{k}=\gamma_{k}^{S D}=\left(g_{k}, g_{k}\right) /\left(A g_{k}, g_{k}\right)$. The method of Minimum Residues [5] (shortly, MR) chooses $\gamma_{k}$ that minimizes $r_{k}$, which gives $\gamma_{k}=\gamma_{k}^{M R}=\left(A g_{k}, g_{k}\right) /\left(A^{2} g_{k}, g_{k}\right)$. Both methods only look one-step forward. The method of Conjugate Gradients (CG in what follows) minimizes $R_{k}^{\prime}=\prod_{j=0}^{k-1} r_{j}^{\prime}$ with respect to the sequence $\gamma_{0}, \gamma_{1}, \ldots, \gamma_{k-1}$ and the method of Conjugate Residuals [3, p.547] (CR) does the same with $R_{k}$; we shall denote $R_{k}^{C R}=\min _{\gamma_{0}, \ldots, \gamma_{k-1}} R_{k}$. The construction of CG and CR does not require the direct minimization of $R_{k}^{\prime}$ or $R_{k}$ with respect to the step-sizes $\gamma_{j}$, but an implementation of the Gram-Schmidt orthogonalization process leads to the construction of the optimal polynomial $P_{k}(\lambda)$ which minimizes either $R_{k}^{\prime}$ or $R_{k}$. In this paper, we shall focus on the rate $R_{k}$ and our competitor will therefore be CR.

Using the expression (5) below for the rate $R_{k}$, we have $R_{k} \leq \max _{\lambda \in[m, M]} P_{k}^{2}(\lambda)$, where $P_{k}(\lambda)$ is a polynomial of degree $k$ satisfying $P_{k}(0)=1$. The right-hand side of the last inequality is minimized for $P_{k}=P_{k}^{*}$, where

$$
P_{k}^{*}(\lambda)=c_{k} T_{k}\left(\frac{2 \lambda-(M+m)}{M-m}\right), \quad c_{k}=T_{k}^{-1}\left(\frac{\rho+1}{\rho-1}\right)
$$

and $T_{k}(\cdot)$ is the $k$-th Chebyshev polynomial of the first kind: $T_{k}(t)=\cos [k \arccos (t)]$. Hence, the rate $R_{k}^{C R}$ of the $\mathrm{CR}$ algorithm satisfies $R_{k}^{C R} \leq R_{k}^{*}$, where

$$
R_{k}^{*}=c_{k}^{2}=\frac{1}{4}\left(R_{\infty}^{k / 2}+R_{\infty}^{-k / 2}\right)^{-2} \text { and } R_{\infty}=\lim _{k \rightarrow \infty}\left(R_{k}^{*}\right)^{1 / k}=\left(\frac{\sqrt{\rho}-1}{\sqrt{\rho}+1}\right)^{2}
$$

There are no iterative methods of the form (2) that achieve smaller values of the rate $R_{k}$ than $R_{k}^{C R}$, the rate of CR within $k$ iterations. Therefore, our competition with CR cannot be in terms of $R_{k}$ only and, in addition to the rate of convergence, we shall consider the complexity of the 
methods, which will be measured by the number of inner products computed after $k$ iterations. The purpose of the paper is to present a method that requires less computations than CR and at the same time achieves asymptotically the same rate as the worst-case convergence rate of CR when stopped at some iteration $k<n$ (when $n$ is large, the asymptotic regime may be reached well before $n$ iterations). As will be seen in (19), for any $k>1$ the proposed method requires no more than $C \log k+4$ computations of inner products $(C \simeq 8.31)$ in $k$ iterations which, for large $k$, is much smaller than the $2 k$ computations of inner products required by $\mathrm{CR}$ and $\mathrm{CG}$. This makes our method competitive when the matrix $A$ in (1) is sparse and inner products yield the main contribution to the computational cost. For instance, in parallel computing the computation of inner products requires a global communication between processors, the price of which increases as the number of processors grows, whereas matrix by vector multiplications only require local communication between neighboring processors, see [12, Sect. 4.4].

The paper is organized as follows. In Sect. 2 we introduce some mathematical formalism and formulate several auxiliary statements needed to explain the construction and properties of the proposed method. In Sect. 2 we also explains how $\max _{x_{0}, A}\left(R_{k}\right)^{1 / k}$ can be made arbitrary close to its lower bound by using step-sizes $\gamma_{k}$ that are suitably distributed. In Sect. 3 we construct an auxiliary sequence $\left\{z_{j}\right\}$ which is one of the main ingredients for the construction of the main algorithm in Sect. 4. In Sect. 3 and 4 we also explain the algorithm and validate its properties. Numerical examples are discussed in Sect. 5

\section{Preliminary results}

Decompose the initial vector $g_{0}$ in the basis of eigenvectors $\left\{q_{1}, \ldots, q_{n}\right\}: g_{0}=\sum_{i=1}^{n} \alpha_{i} q_{i}$. Then for all $k \geq 1$ we have the decompositions $g_{k}=\sum_{i=1}^{n} \alpha_{i} P_{k}\left(\lambda_{i}\right) q_{i}$. The squared $L_{2}$-norm of $g_{0}$ is $\left\|g_{0}\right\|^{2}=\left(g_{0}, g_{0}\right)=\sum_{i=1}^{n} \alpha_{i}^{2}$ and the squared $L_{2}$-norm of $g_{k}$ is $\left\|g_{k}\right\|^{2}=\left(g_{k}, g_{k}\right)=$ $\sum_{i=1}^{n} \alpha_{i}^{2} P_{k}^{2}\left(\lambda_{i}\right)$. Using these representations we can write the rate $R_{k}$ as

$$
R_{k}=\frac{\left(g_{k}, g_{k}\right)}{\left(g_{0}, g_{0}\right)}=\left[\sum_{i=1}^{n} \alpha_{i}^{2} P_{k}^{2}\left(\lambda_{i}\right)\right] / \sum_{i=1}^{n} \alpha_{i}^{2}=\sum_{i=1}^{n} p_{i}^{(0)} P_{k}^{2}\left(\lambda_{i}\right)
$$

where $p_{i}^{(0)}=\alpha_{i}^{2} / \sum_{j=1}^{n} \alpha_{j}^{2} \geq 0$ and $\sum_{i=1}^{n} p_{i}^{(0)}=1$. Without loss of generality all $\alpha_{i}^{2}$ can be assumed to be strictly positive. Indeed, if $\alpha_{i}=0$ for some $i$ then the matrix $A=\sum_{j=1}^{n} \lambda_{j} q_{j} q_{j}^{T}$ can be replaced with $\tilde{A}=\sum_{j \neq i} \lambda_{j} q_{j} q_{j}^{T}$. The equality $\alpha_{i}=0$ would mean that the projection of $\lambda_{i} x_{0}$ (and therefore of all $\lambda_{i} x_{k}, k \geq 0$ ) to the vector $q_{i}$ is equal to $\left(b, q_{i}\right)$, the projection of $b$ to $q_{i}$.

The projection of the vector $g_{k}$ on the eigenvector $q_{i}$ is $\left(g_{k}, q_{i}\right)=\alpha_{i} P_{k}\left(\lambda_{i}\right)$. We define $p_{i}^{(k)}=\left(g_{k}, q_{i}\right)^{2} /\left(g_{k}, g_{k}\right)=\alpha_{i}^{2} P_{k}^{2}\left(\lambda_{i}\right) / \sum_{j=1}^{n} \alpha_{j}^{2} P_{k}^{2}\left(\lambda_{j}\right)$ and interpret this as a point mass at $\lambda_{i}$. Then, the measure $\nu_{k}$ defined by its masses $\nu_{k}\left(\lambda_{i}\right)=p_{i}^{(k)}$ at $\lambda=\lambda_{i}(i=1, \ldots, n)$ characterizes the normalized gradient $g_{k} /\left\|g_{k}\right\|$.

For any real $\alpha$, define $\mu_{\alpha}^{(k)}$ as the $\alpha$-th moment of the probability measure $\nu_{k}: \mu_{\alpha}^{(k)}=$ $\sum_{i=1}^{n} \lambda_{i}^{\alpha} p_{i}^{(k)}=\left(A^{\alpha} g_{k}, g_{k}\right) /\left(g_{k}, g_{k}\right)$. Using the main iteration (2), we obtain the following up- 
dating formula for the measure $\nu_{k+1}$ :

$$
p_{i}^{(k+1)}=\nu_{k+1}\left(\lambda_{i}\right)=\frac{\alpha_{i}^{2} P_{k+1}^{2}\left(\lambda_{i}\right)}{\left(g_{k+1}, g_{k+1}\right)}=\frac{\alpha_{i}^{2}\left(1-\gamma_{k} \lambda_{i}\right)^{2} P_{k}^{2}\left(\lambda_{i}\right)}{\left(g_{k+1}, g_{k+1}\right)}=\frac{\left(1-\gamma_{k} \lambda_{i}\right)^{2} p_{i}^{(k)}}{r_{k}},
$$

where

$$
r_{k}=\frac{\left(g_{k+1}, g_{k+1}\right)}{\left(g_{k}, g_{k}\right)}=\frac{\left(g_{k}, g_{k}\right)-2 \gamma_{k}\left(A g_{k}, g_{k}\right)+\gamma_{k}^{2}\left(A^{2} g_{k}, g_{k}\right)}{\left(g_{k}, g_{k}\right)}=1-2 \gamma_{k} \mu_{1}^{(k)}+\gamma_{k}^{2} \mu_{2}^{(k)} .
$$

The following two results will be useful for the construction of the main algorithm and for evaluating its performance.

Proposition 1 (Monotonicity) The condition $r_{k}<1$ is equivalent to

$$
\beta_{k}>\mu_{2}^{(k)} /\left(2 \mu_{1}^{(k)}\right) .
$$

Proof Follows from (7) and $\beta_{k}=1 / \gamma_{k}$.

Proposition 2 (Moment inequalities) Let $\nu$ be any probability measure on $[m, M]$ and denote by $\mu_{\alpha}$ the $\alpha$-th moment of $\nu: \mu_{\alpha}=\mu_{\alpha}(\nu)=\int_{m}^{M} t^{\alpha} \nu(d t)$. Then

$$
m \leq \mu_{1} \leq \frac{\mu_{2}}{\mu_{1}} \leq \frac{\mu_{3}}{\mu_{2}} \leq \frac{\mu_{4}}{\mu_{3}} \leq \cdots \leq M .
$$

Proof The Cauchy-Schwarz inequality implies $\mu_{\alpha+2} \mu_{\alpha} \geq\left(\mu_{\alpha+1}\right)^{2}$ for any $\alpha$. Moreover, for all $t \in[m, M]$ we have $t(M-t) \geq 0$ so that $\int_{m}^{M} t^{\alpha}(M-t) \nu(d t)=M \mu_{\alpha}-\mu_{\alpha+1} \geq 0$; that is, $\mu_{\alpha+1} / \mu_{\alpha} \leq M$. Similarly, $m \leq \mu_{\alpha+1} / \mu_{\alpha}$. This implies (9).

For any choice of the step-size sequence $\left\{\gamma_{k}\right\}$ in (2) the rate $R_{k}$ satisfies $\max _{x_{0}, A} R_{k} \geq R_{k}^{*}$ for $k<n$, with $\left(R_{k}^{*}\right)^{1 / k}$ decreasing to $R_{\infty}$ as $k \rightarrow \infty$, see (4). Our objective is to construct simple gradient algorithms such that $R_{k}^{1 / k}$ gets close to the bound $R_{\infty}$. If the values $m$ and $M$ were known, the results of [9] show that this can easily be achieved using some predefined sequences of step-sizes $\gamma_{k}$, without any need for computation of inner products. For example, one can use $\beta_{j}=1 / \gamma_{j}$ as the roots of the polynomials (3), but the degree of the polynomial must then be pre-specified (and be equal to the number of iterations to be performed); the ordering of the roots is important too.

One of the main points in our argumentation uses the fact that by a suitable choice of the coefficients $\gamma_{0}, \gamma_{1}, \ldots$ we can easily force the asymptotic relation $\nu_{k}(\lambda) \rightarrow 0$ as $k \rightarrow \infty$ for all $\lambda \in(m, M)$. That is, for large $k$ we can force the projections of the normalized gradient $g_{k} /\left\|g_{k}\right\|$ to all eigenvectors except for $q_{1}$ and $q_{n}$ to be negligible. This happens, for example, if the sequence $\left\{\beta_{k}\right\}$ is concentrated in an interval $\left[m^{\prime}, M^{\prime}\right]$ with $m<m^{\prime}<M^{\prime}<M$ and the values of $\left\{\beta_{k}\right\}$ are well-spread over $\left[\mathrm{m}^{\prime}, M^{\prime}\right]$. More precisely, the following result is a particular case of Theorem 1 in [9].

Proposition 3 (Convergence to the set of measures supported at two points) $A s$ sume that $\beta_{k} \in\left[m^{\prime}, M^{\prime}\right]$ with $m<m^{\prime} \leq M^{\prime}<M$ for all $k$ and that the sequence $\left\{\beta_{k}\right\}$ has an asymptotic distribution function $F(t)$ such that $\int \log (t-\lambda)^{2} d F(t)<\max \left\{\int \log (M-\right.$ $\left.t)^{2} d F(t), \int \log (t-m)^{2} d F(t)\right\}$ for all $\lambda \in(m, M)$. Then the algorithm (2) associated with the sequence $\left\{\beta_{k}\right\}$ is such that $\lim _{k \rightarrow \infty} \nu_{k}\left(\lambda_{i}\right)=0$ for all $i=2, \ldots, n-1$. 
The following statement is a simple consequence of Proposition 3.

Proposition 4 (Attraction to one-point measures) Under the conditions of Proposition 3, the sequence of measures $\nu_{k}$ attracts to the set of two-point measures supported on $\{m, M\}$. If, moreover, the asymptotic distribution of the sequence $\left\{\beta_{k}\right\}$ is biased towards $m$ (respectively $M$ ), then the sequence $\left\{\nu_{k}\right\}$ tends to the one-point measure concentrated at $M$ (respectively $m$ ). This happens in particular when the sequence $\left\{\beta_{k}\right\}$ is generated by pairs as in Proposition 4 with $\beta_{2 j+1}=M^{\prime}+m^{\prime}-\beta_{2 j}$ for all $j \geq j_{0}, m^{\prime}=m+\varepsilon_{m}, M^{\prime}=M-\varepsilon_{M}$ and $\varepsilon_{m} \neq \varepsilon_{M}$.

Once the attraction of the sequence $\left\{\nu_{k}\right\}$ to the set of measures supported at the two-point set $\{m, M\}$ is obtained, it is enough to consider the rate of convergence $r_{k}$ obtained for the two-point measures and the following is true, see [9, Th. 2].

Proposition 5 (Asymptotic rate) Assume that the conditions of Proposition 3 are satisfied and the values $\beta_{k}=1 / \gamma_{k}$ are generated by symmetric pairs for large $k$; that is, $\beta_{2 j+1}=M+$ $m-\beta_{2 j}$ for all $j \geq j_{0}$. Then the asymptotic rate $R$ is $R=\exp \left\{2 \int[\log (t-m)-\log t] d F(t)\right\}$.

If the limiting distribution function $F(\cdot)$ has the arcsine density on $\left[m^{\prime}, M^{\prime}\right]$

$$
p(t)=\frac{1}{\pi \sqrt{\left(t-m^{\prime}\right)\left(M^{\prime}-t\right)}}, \quad m^{\prime} \leq t \leq M^{\prime}
$$

with $m^{\prime}=m+\varepsilon$ and $M^{\prime}=M-\varepsilon$ for some small $\varepsilon \geq 0$, then, as shown in [9], the asymptotic rate of convergence is $R_{\varepsilon}=R_{\infty}(1+4 \sqrt{\varepsilon(M-m)})+O(\varepsilon), \varepsilon \rightarrow 0$.

Numerical results in [9] confirm the observation that if the asymptotic distribution of the sequence $\left\{\beta_{k}\right\}$ is close to the distribution with the arcsine density on $[m, M]$, then the asymptotic rate of the algorithm (2) is close to $R_{\infty}$. Furthermore, we observed that for the BarzilaiBorwein (BB) [1] as well as many other gradient algorithms we have investigated (see, e.g., [4]), when the rate $R_{k}^{1 / k}$ approached $R_{\infty}$ then the distribution of the sequence $\left\{\beta_{k}\right\}$ was close to the distribution with the arcsine density on $[m, M]$. This phenomena can be related the fact that, roughly speaking, for large $k$ the roots of all orthogonal polynomials behave similarly. In particular, if a probability measure $\nu_{0}$ has a density $p_{0}(\cdot)$ with respect to the Lebesgue measure on $[m, M]$, with $p_{0}(t)>0$ except for a set of zero Lebesgue measure, then the roots of the orthogonal polynomials $P_{k}^{*}$ with respect to $\nu_{0}$ are distributed asymptotically according to the arcsine density on $[m, M]$, see $[2$, Th. XIII]. As any efficient gradient algorithm should not deviate much from the behaviour of the optimal algorithm (which locates $\beta_{k}$ at the roots of the optimal polynomials orthogonal with respect to $\left.\nu_{0}\right)$, the asymptotic distribution of $\left\{\beta_{k}\right\}$ for this algorithm should be close to the arcsine distribution on $[m, M]$.

\section{Numerical sequence for generating step-sizes}

The method presented below generates step-sizes $\gamma_{k}$ such that the inverse sequence $\left\{\beta_{k}\right\}=$ $\left\{1 / \gamma_{k}\right\}$ has the arcsine distribution on a given interval $\left[m^{\prime}, M^{\prime}\right]$, where $m^{\prime}$ and $M^{\prime}$ can be chosen as $m^{\prime}=m+\varepsilon$ and $M^{\prime}=M-\varepsilon$, with small $\varepsilon \geq 0$. In practice $m$ and $M$ are unknown and we shall use estimates $m^{\prime}=\widehat{m}_{k}$ and $M^{\prime}=\widehat{M}_{k}$ of $m$ and $M$, with $m<\widehat{m}_{k}<\widehat{M}_{k}<M$. 
We shall initiate the algorithm (2) by using two iterations of MR, that is with $\beta_{0}=\mu_{2}^{(0)} / \mu_{1}^{(0)}$ and $\beta_{1}=\mu_{2}^{(1)} / \mu_{1}^{(1)}$; this provides us with $\widehat{m}_{2}=\min \left\{\beta_{0}, \beta_{1}\right\}$ and $\widehat{M}_{2}=\max \left\{\beta_{0}, \beta_{1}\right\}$, the initial estimates of $m$ and $M$.

The following iterations will use the sequence $\left\{z_{j}\right\}$ in $[0,1]$ introduced below: to compute $\beta_{k}$ we rescale the values of $z_{j}$ to $\left[\mathrm{m}^{\prime}, M^{\prime}\right]$ through

$$
\beta_{k}=m^{\prime}+\left(M^{\prime}-m^{\prime}\right) z_{j}, \text { for } k=2,3, \ldots \text { and some } j=j(k) .
$$

Note that if $m^{\prime}$ and $M^{\prime}$ are fixed, $\left\{z_{j}\right\}$ has the asymptotic density

$$
q(z)=1 /[\pi \sqrt{z(1-z)}], 0 \leq z \leq 1,
$$

$j \leq k$ and $j / k \rightarrow 1$ as $k \rightarrow \infty$, then the sequence $\left\{\beta_{k}\right\}$ constructed by (11) has the asymptotic density (10).

A discussion concerning the choice of $\left\{z_{j}\right\}$ with density (12) is contained in [9]. We have experimented with a number of sequences and provide below one of the best if not the best.

The sequence $\left\{\mathbf{z}_{\mathbf{j}}\right\}$. For all $j \geq 0$, we define $v_{j}=\{\varphi(j+1)\}$, where $\varphi=(\sqrt{5}+1) / 2 \simeq$ $1.61803 \ldots$ and $\{a\}$ denotes the fractional part of $a$. For $j=0,1, \ldots$, set

$$
z_{j}=\left(1+\cos \left(\pi u_{j}\right)\right) / 2 \text {, where } u_{2 j}=\min \left\{v_{j}, 1-v_{j}\right\}, u_{2 j+1}=\max \left\{v_{j}, 1-v_{j}\right\} .
$$

All three sequences above, $\left\{v_{j}\right\},\left\{u_{j}\right\}$ and $\left\{z_{j}\right\}$, belong to $[0,1]$. The asymptotic distribution of $\left\{v_{j}\right\}$ and $\left\{u_{j}\right\}$ is uniform and the asymptotic distribution of $\left\{z_{j}\right\}$ has the arcsine density (12). The sequence $\left\{v_{j}\right\}$ consists of the fractional parts of multiples of the golden ratio $\varphi$, the sequence $\left\{u_{j}\right\}$ is a symmetrized version of $\left\{v_{j}\right\}$ while the sequence $\left\{z_{j}\right\}$ has the required arcsine density in $[0,1]$ and is obtained from $\left\{u_{j}\right\}$ by a suitable transformation.

The reasons behind the proposed choice of the sequence $\left\{z_{j}\right\}$ are the following:

- simplicity of construction and analysis;

- required asymptotic distribution (with empirical distribution converging fast to the limiting distribution - a consequence of the good uniformity of $\left.\left\{v_{j}\right\}\right)$;

- symmetry $z_{2 j+1}=1-z_{2 j}$ and $z_{2 j}>\frac{1}{2}$ for all $j$.

A benefit of using symmetric points is that by choosing the largest $z_{j}$ (and therefore the largest $\beta_{k}$ ) first in each symmetric pair one makes the behavior of the proposed algorithm more monotonic. Indeed, $\beta_{k} \geq(M+m) / 2$ implies $\beta_{k}>M / 2 \geq \mu_{2}^{(k)} /\left(2 \mu_{1}^{(k)}\right)$, see $(9)$, and therefore $r_{k}<1$, see (8). Moreover, (6) indicates that when $\beta_{k}=1 / \gamma_{k}$ is large, the measure $\nu_{k+1}$ allocates more weight than $\nu_{k}$ to small eigenvalues $\lambda_{i}$, so that $\mu_{2}^{(k+1)} /\left(2 \mu_{1}^{(k+1)}\right)$ tends to be small and the next iteration is likely to be monotonic too according to (8).

For the sequence $z_{0}, z_{1}, z_{2}, \ldots$ constructed above we define the sequences of record moments $L_{\min }=\left\{L_{\min }(j)\right\}_{j=0}^{\infty}$ and $L_{\max }=\left\{L_{\max }(j)\right\}_{j=0}^{\infty}$ as follows: $L_{\min }(0)=L_{\max }(0)=0$ and $L_{\min }(j+$ $1)=\min \left\{k>L_{\min }(j): z_{k}<z_{L_{\min }(j)}\right\}, L_{\max }(j+1)=\min \left\{k>L_{\max }(j): z_{k}>z_{L_{\max }(j)}\right\}$ for $j \geq 0$. These two sequences of record moments are $L_{\min }=\{0,1,3,5,9,15, \ldots\}$ and $L_{\max }=$ $\{0,2,4,8,14, \ldots\}$ with $L_{\min }(j+1)=L_{\max }(j)+1$ for $j=0,1, \ldots$

The following statement expresses the sequences $L_{\min }$ and $L_{\max }$ in terms of the Fibonacci numbers. Recall the sequence of Fibonacci numbers $\left\{F_{N}\right\}_{N=1}^{\infty}=\{1,1,2,3,5,8,13,21,34 \ldots\}$ and the exact formula $F_{N}=\left(\varphi^{N}-\widehat{\varphi}^{N}\right) / \sqrt{5}$, where $\varphi$ is the golden ratio and $\widehat{\varphi}=-1 / \varphi$. 
Proposition 6 We have $L_{\max }(j)=2\left(F_{j+2}-1\right)$ for $j=0,1, \ldots$ and $L_{\min }(j)=2 F_{j+1}-1$ for $j=1,2, \ldots$ with $L_{\min }(0)=0$, where $F_{i}$ is the $i$-th Fibonacci number.

Proof The statement is a direct consequence of the following two classical results of the theory of Diophantine approximations: (i) for the sequence $\{k \alpha\}$ with any irrational $\alpha$, the successive minimal and maximal values occur when $k=q$ in the denominator of a convergent $p / q$ for $\alpha$ in the standard continued fraction expansion of $\alpha$, see [11]; (ii) the convergents of $\alpha=\varphi-1$ are $F_{j} / F_{j+1}$ for $j>1$.

Proposition 7 Let $\left\{z_{j}\right\}$ be the sequence constructed in (13) and $\delta_{j}$ be the number of upper record moments appearing among $z_{0}, z_{1}, \ldots, z_{j}$; that $i s$,

$$
\delta_{j}=\left\{\text { number of } i \geq 0 \text { s.t. } L_{\max }(i) \leq j\right\}=1+\max \left\{i \geq 0 \text { : s.t. } L_{\max }(i) \leq j\right\} .
$$

Then for all $j>1$ we have the inequality $\delta_{j}<c \log j$ and the asymptotic relation $\delta_{j}=$ $c \log j+O(1)$ as $j \rightarrow \infty$, where $c=1 / \log (\varphi) \simeq 2.078$.

Proof We have $L_{\max }(i)=2\left(F_{i+2}-1\right)$ for $i=0,1, \ldots$ This gives for all $j>1$ :

$$
\begin{aligned}
\delta_{j}-1= & \max \left\{i: 2\left(F_{i+2}-1\right) \leq j\right\}=\max \left\{i: F_{i+2} \leq j / 2+1\right\} \\
= & \max \left\{i: \varphi^{i+2}-\widehat{\varphi}^{i+2} \leq \sqrt{5}(j / 2+1)\right\}=\max \left\{i: \varphi^{i} \leq \sqrt{5}(j / 2+1) / \varphi^{2}+\widehat{\varphi}^{i+4}\right\} \\
& <\max \left\{i: \varphi^{i} \leq \sqrt{5} j /\left(2 \varphi^{2}\right)+1\right\}=\max \left\{i: i \leq \log \left(\sqrt{5} j /\left(2 \varphi^{2}\right)+1\right) / \log \varphi\right\} \\
& \leq \log \left(\sqrt{5} j /\left(2 \varphi^{2}\right)+1\right) / \log \varphi=\left[\log j+\log \left(\sqrt{5} /\left(2 \varphi^{2}\right)+1 / j\right)\right] / \log \varphi \\
& <\log j / \log \varphi=c \log j .
\end{aligned}
$$

As $j \rightarrow \infty$, this gives the asymptotic equation (in $x$ ) for $\delta_{j}: 2 \varphi^{x+2}=\sqrt{5} j$, implying $\delta_{j}=$ $c \log j+O(1)$ as $j \rightarrow \infty$.

\section{The algorithm}

Since we assume that the smallest and largest eigenvalues $\lambda_{1}=m$ and $\lambda_{n}=M$ of the matrix $A$ are unknown, these values have to be estimated. Our estimators will be based on the inequalities (9) and we shall use

$$
\widehat{m}_{k+1}=\min \left\{\mu_{1}^{(i)}, i \in I_{k}^{m}\right\} \text { and } \widehat{M}_{k+1}=\max \left\{\mu_{\alpha+1}^{(i)} / \mu_{\alpha}^{(i)}, i \in I_{k}^{M}\right\}
$$

where in the algorithm below $\alpha=3$ and $I_{k}^{m}, I_{k}^{M}$ are subsets of $\{0,1, \ldots, k\}$ which will be determined by the algorithm. The case $\alpha=0$ in (15) has been considered in [9]. The resulting algorithms are efficient but not efficient enough to compete with $\mathrm{CR}$ and $\mathrm{CG}$. The value $\alpha=3$ in (15) leads to much better estimates of $M$ and hence to much more cost-efficient algorithms which use very thin sets $I_{k}^{m}$ and $I_{k}^{M}$ and as a consequence few inner products to compute. Notice that from Proposition 2 we have $m \leq \widehat{m}_{k}$ and $\widehat{M}_{k} \leq M$ for all $k$, as required by Proposition 3 .

Using good estimators of $M$ is important for the following reason. Generating the $\beta_{k}=1 / \gamma_{k}$ by pairs and using the largest first in each pair favors the estimation of $m$ against that of $M$. Indeed, the measure $\nu_{k}$ attracts to the set of two-point measures supported at $\{m, M\}$. For a 
measure $\nu_{2 i}$ in this set, two iterations of (6) with $\beta_{2 i+1}=M+m-\beta_{2 i}$ give $\nu_{2 i+2}=\nu_{2 i}$ and thus $\mu_{\alpha}^{(2 i+2)}=\mu_{\alpha}^{(2 i)}$ for all $\alpha$. This means that asymptotically the estimation of $m$ and $M$ can only be improved for even values of $j$ only, where $j$ is the index of the sequence $\left\{z_{j}\right\}$. Using the largest $\beta$ first in a pair yields $\beta_{2 i}>(M+m) / 2$ resulting in a small $\mu_{1}^{(2 i+1)}$ that may improve the current estimation of $m$ when $\beta_{2 i}$ is close to $M$. In view of Proposition 4 , this better estimation of $m$ than $M$ results in the concentration of $\nu_{k}$ at $M$. Since in this case the ratio $\mu_{2}^{(k)} /\left(2 \mu_{1}^{(k)}\right)$ becomes close to $M / 2$ when $M / m$ is large, the monotonicity condition (8) would be violated frequently. In the algorithm proposed below we avoid this by using a good estimator of $M$.

Monotonicity can be imposed by forcing the measures $\nu_{k}$ to be concentrated more at $m$ rather than at $M$ when needed. Indeed, the condition (8) is always satisfied when $\mu_{2}^{(k)} /\left(2 \mu_{1}^{(k)}\right)$ is close to $m / 2$ since $\beta_{k}$ is larger than $\widehat{m}_{k}>m$. This movement of the masses is achieved by using a step with large $\beta_{k}$ when $\nu_{k-1}$ is close to the delta measure at $M$. In practice, we simply use $\beta_{k}=\widehat{M}_{k}$ when we observe $\widehat{M}_{k}>\widehat{M}_{k-1}$.

The calculation of $\mu_{1}^{(k)}$ needed for the computation of $\widehat{m}_{k+1}$ does not require the calculation of $A g_{k}$ at step $k$. Indeed, allowing a delay of one step in the estimation of $m,(2)$ gives $A g_{k}=$ $\beta_{k}\left(g_{k}-g_{k+1}\right)$, so that

$$
\mu_{1}^{(k)}=\frac{\left(A g_{k}, g_{k}\right)}{\left(g_{k}, g_{k}\right)}=\beta_{k}\left[1-\frac{\left(g_{k}, g_{k+1}\right)}{\left(g_{k}, g_{k}\right)}\right]
$$

Allowing a delay of two iterations, we get $A^{2} g_{k-1}=\beta_{k-1} \beta_{k}\left(g_{k+1}-g_{k}\right)+\beta_{k-1}^{2}\left(g_{k-1}-g_{k}\right)$ and $A g_{k-1}=\beta_{k-1}\left(g_{k-1}-g_{k}\right)$ when using (2). This gives

$$
\frac{\mu_{4}^{(k-1)}}{\mu_{3}^{(k-1)}}=\frac{\left(A^{2} g_{k-1}, A^{2} g_{k-1}\right)}{\left(A^{2} g_{k-1}, A g_{k-1}\right)}=\beta_{k-1}+\beta_{k} \frac{\left(\beta_{k}\left(g_{k+1}-g_{k}\right)+\beta_{k-1}\left(g_{k-1}-g_{k}\right), g_{k+1}-g_{k}\right)}{\left(\beta_{k}\left(g_{k+1}-g_{k}\right)+\beta_{k-1}\left(g_{k-1}-g_{k}\right), g_{k-1}-g_{k}\right)} \text {. }
$$

This avoids the use of additional matrix-vector multiplications while computing the estimates of $m$ and $M$. The calculations of inner products required for the update of the estimators of $m$ and $M$ can be done in parallel. Moreover, these estimators need to be updated at iteration $k$ only when the value $z$ used at previous iteration was larger than the maximum over all previous $z_{i}$. The updating rule is

$$
\widehat{m}_{k+1}=\min \left\{\widehat{m}_{k}, \mu_{1}^{(k)}\right\} \text { and } \widehat{M}_{k+1}=\max \left\{\widehat{M}_{k}, \mu_{4}^{(k-1)} / \mu_{3}^{(k-1)}\right\}
$$

where $\mu_{1}^{(k)}$ and $\mu_{4}^{(k-1)} / \mu_{3}^{(k-1)}$ are computed by (16) and (17) respectively.

We are now ready to formulate the main algorithm; its MATLAB implementation is available at http://www.i3s.unice.fr/ pronzato/Matlab/goldenArcsineQ.m

\section{Algorithm.}

\section{Stage I (initialization).}

I.1 Choose $x_{0}$.

I. 2 Choose $\epsilon>0$ used in the stopping rule.

I.2 For $k=0,1$ set $x_{k+1}=x_{k}-\gamma_{k} g_{k}$ and $g_{k+1}=A x_{k+1}-b$, where $\gamma_{k}=\left(A g_{k}, g_{k}\right) /\left(A g_{k}, A g_{k}\right)$.

I.3 Compute $\widehat{m}_{2}=\min \left\{1 / \gamma_{0}, 1 / \gamma_{1}\right\}$ and $\widehat{M}_{2}=\max \left\{1 / \gamma_{0}, 1 / \gamma_{1}\right\}$.

I. 4 Set $j_{0}=-1, j_{1}=1$.

I.5 Set $k=2$ and $j=0$. 
Stage II (iterations).

II.1 If $j-1=j_{1}$ and $\widehat{M}_{k}>\widehat{M}_{k-1}$ then set $\beta_{k}=\widehat{M}_{k}$.

Otherwise set $\beta_{k}=\widehat{m}_{k}+\left(\widehat{M}_{k}-\widehat{m}_{k}\right) z_{j}$ and $j \leftarrow j+1$.

II.2 Set $x_{k+1}=x_{k}-\left(1 / \beta_{k}\right) g_{k}$ and $g_{k+1}=A x_{k+1}-b$.

II.3 If $j=j_{0}+j_{1}+2$ then compute $\widehat{m}_{k+1}$ and $\widehat{M}_{k+1}$ using formula (18), check the stopping rule $\left(g_{k}, g_{k}\right) \leq \epsilon$ and set $j_{0} \leftarrow j_{1}, j_{1} \leftarrow j-1$. Otherwise set $\widehat{m}_{k+1}=\widehat{m}_{k}, \widehat{M}_{k+1}=\widehat{M}_{k}$.

II.4 Set $k \leftarrow k+1$ and return to Step II.1.

Note an alternative way of writing the condition $j=j_{0}+j_{1}+2$ in Step II. 3 of the algorithm: $j-2 \in L_{\max }$ where $L_{\max }=\left\{2 F_{i+2}-2: i=0,1, \ldots\right\}=\{0,2,4,8,14, \ldots\}$.

The algorithm uses the common stopping rule $\left(g_{k}, g_{k}\right)<\epsilon$ for some given $\epsilon$. The values of $\left(g_{k}, g_{k}\right)$ are available at the iterations $k$ such that $j-2 \in L_{\max }$. At these iterations, we can check the stopping rule directly. In addition, as we have an (updated) under-estimate for $\rho$ and hence and under-estimate of $R_{\infty}$ given by (4), we can estimate the number of iterations still remaining to achieve the required precision.

The proposed algorithm only requires one matrix-vector multiplication per iteration (used to calculate the gradient $g_{k}=A x_{k}-b$ ), like other gradient methods, and Krylov-space based algorithms, like CR and CG. When $A$ is sparse, the computation of inner products also contributes significantly to the total computational cost. When using parallel computing, it may even yield the main contribution to the computational cost, see [12, Sect. 4.4].

The standard formulation of CG (and also CR) requires the computation of two inner products per iteration. In some sophisticated versions of CG, these two inner products can be computed in parallel, at the possible cost of a slight increase of storage and maybe reduced numerical stability, see for instance $[6,10]$.

The following theorem establishes the upper bound and the asymptotical expression for the total number of inner products computed by the proposed algorithm.

Proposition 8 Let $N_{k}$ be the total number of inner products computed within $k+1$ steps of the proposed algorithm. Then for all $k>0$ we have

$$
N_{k}<4+4 \log k / \log (\varphi) \cong 4+8.31 \log k \text { and } N_{k}=4 \log k / \log (\varphi)+O(1) \text { as } k \rightarrow \infty .
$$

Proof The proposed algorithm requires the computation of four inner products in the initial two iterations and four inner products each time the estimates $\widehat{m}_{k}$ and $\widehat{M}_{k}$ are updated. This is done when $j-2 \in L_{\max }$. Therefore, the total number of inner products computed within $k+1$ steps of the proposed algorithm is equal to $N_{k}=4+4 \delta_{j}$ where $j=j(k)$ is defined by the algorithm and $\delta_{j}$ is defined in (14). Proposition 7 implies that $j(k)<k, \delta_{j}<\log j / \log (\varphi)$ for all $j>1$ and $\delta_{j}=\log j / \log (\varphi)+O(1)$ as $j \rightarrow \infty$. This and the fact $k / j(k) \rightarrow 1$ as $k \rightarrow \infty$ imply (19).

\section{Numerical performance and comparison with other methods}

In order to illustrate the efficiency of the proposed algorithm we consider two test problems. In both problems, we set $m=1, M=1000, n=1000, b=A c$ with $c$ uniformly distributed on the unit sphere. Notice that any increase of $n$ would make the situation more favorable to the algorithm we propose relative to CR and CG. Indeed, for given $m$ and $M$, the behaviors of CR 
and CG depend on the particular values taken by the eigenvalues of $A$ (and generally the rates of CR and CG deteriorate when $n$ increases) whereas the proposed algorithm behaves similarly whatever the dimension $n$ and the spectrum of $A$.

In Problem $1, x_{0}$ is uniformly distributed on the unit sphere and the $n$ normalized eigenvalues are distributed according to the Marchenko-Pastur density $p_{c}(x)=\sqrt{(b-x)(x-a)} /\left(2 \pi x c^{2}\right)$, $a=(1-c)^{2}<x<b=(1+c)^{2}$, where we chose a 'neutral' value $c=1 / 2$. These random eigenvalues are then scaled from the interval $[a, b]=[1 / 4,9 / 4]$ back to $[m, M]=[1,1000]$. The Marchenko-Pastur density describes the asymptotic behavior of eigenvalues of suitably randomized large positive definite matrices. Problem 2 corresponds to the worst-case situation for $n-1$ steps of CR: the eigenvalues of the matrix $A$ are $\lambda_{i}=\frac{M+m}{2}+\frac{M-m}{2} \cos \left(\frac{\pi i}{n-1}\right)$ for $i=$ $1, \ldots, n$ and the initial point $x_{0}$ is such that the $\alpha_{i}^{2}$ in the decomposition $g_{0}=\sum_{i} \alpha_{i} q_{i}$ are proportional to $\alpha_{1}^{2}=1 / 2 \lambda_{1}, \alpha_{n}^{2}=1 / 2 \lambda_{n}$ and $\alpha_{j}^{2}=1 / \lambda_{j}$ for $j=2, \ldots, n-1$, see [8] for details.

In Fig. 1 and 2 for typical runs of SD, CR and the proposed algorithm (abbreviated as 'Alg') we plot the evolution of the decimal logarithm of the $L_{2}$-norm $\left\|g_{k}\right\|=\sqrt{\left(g_{k}, g_{k}\right)}$ of the gradients.

(a)

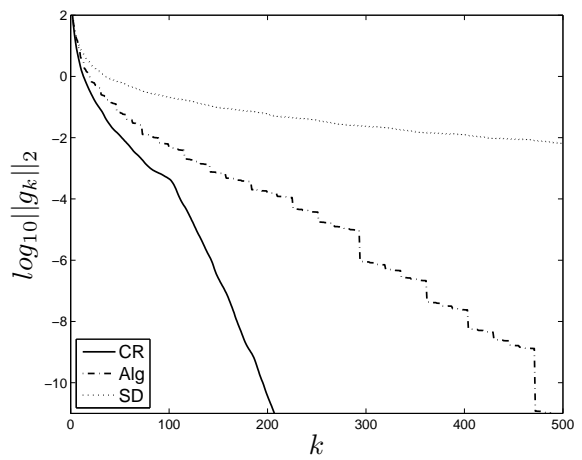

(b)

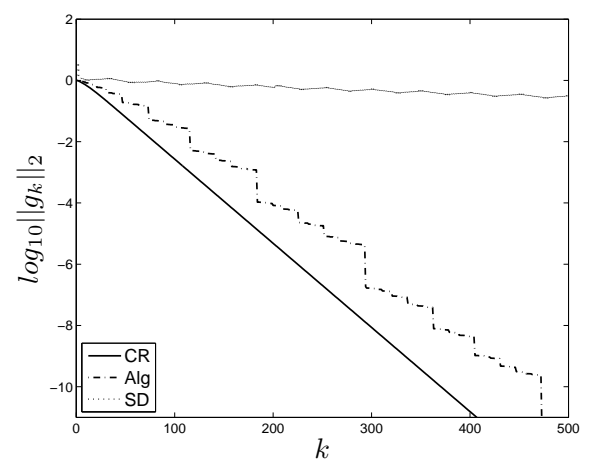

Fig. $1 \log _{10}\left\|g_{k}\right\|$ against the number of iterations $k$ for Problem 1 (left) and Problem 2 (right).

CR is optimal in terms of the rate of convergence $R_{k}$ after $k$ iterations, the proposed algorithm cannot therefore do better with respect to this criterion. It indeed does worse, see Fig. 1. Nevertheless, although the proposed algorithm requires more iterations than CR to obtain a similar value of $\left\|g_{k}\right\|$, it is very competitive in terms of the computational cost. To illustrate this, consider again Problem 1 as in Fig. 1a. Fig. 2a shows $\log _{10}\left\|g_{k}\right\|$ against the number of iterations where inner products are computed (possibly in parallel). Fig. 2b shows $\log _{10}\left\|g_{k}\right\|$ against the total number of inner products calculated. For CR, the plot on Fig. 2a exactly matches that on Fig. 1a as we assumed that the computations of inner products are parallelized at each iteration; the decrease of $\log _{10}\left\|g_{k}\right\|$ for CR is two times slower on Fig. 2b than on Fig. 1a since CR uses two inner products per iteration.

Note that in the run of 500 iterations of the proposed algorithm, the estimators for $m$ and $M$ were updated 12 times (in addition to the computation of the estimators at Stage I) and 3 (resp. 5) iterations with $\beta_{k}=\widehat{M}_{k}$ were used for the Problem 1 (resp. Problem 2). The total number of inner products computed was $52=4+4 \cdot 12$. 
(a)

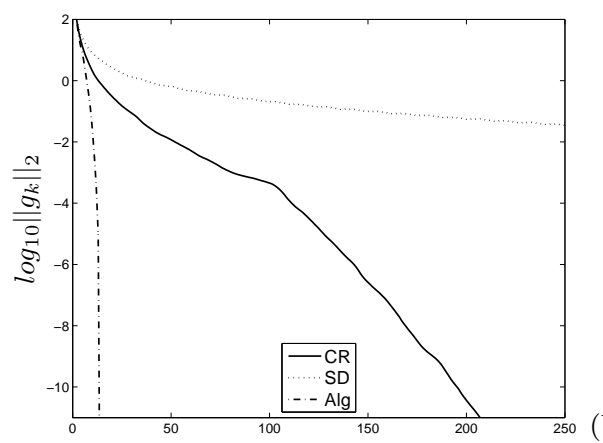

(b)

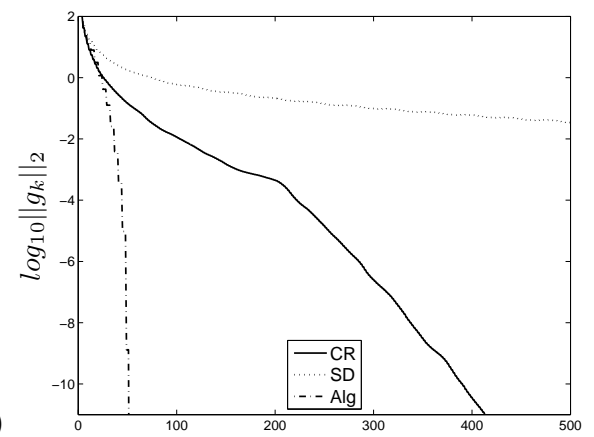

Fig. 2 Problem 1: $\log _{10}\left\|g_{k}\right\|$ against the number of iterations where inner products need to be computed (left) and the total number of inner products (right).

\section{Conclusion}

We proposed a gradient algorithm for optimizing a quadratic function $\frac{1}{2}(A x, x)-(b, x)$, where $A$ is a symmetric positive-definite matrix of size $n \times n$. We have demonstrated that if $n$ is large and the distribution of the eigenvalues of the matrix $A$ is dense on the spectrum, then the convergence rate of the algorithms we propose is not much worse than the rate achieved by the Conjugate-Gradient algorithm, although the iterations are simpler. For sparse matrices, the proposed algorithm can significantly outperform the Conjugate-Gradient algorithm in terms of computational cost: only $O(\log k)$ inner products need to be computed in $k$ iterations.

\section{References}

1. Barzilai, J., Borwein, J.: Two-point step size gradient methods. IMA J. Numer. Anal. 8, 141-148 (1988)

2. Erdös, P., Turan, P.: On interpolation. III. Interpolation theory of polynomials. Ann. Math. 41(3), 510-553 (1940)

3. Golub, G., Loan, C.V.: Matrix Computations, third edn. Johns Hopkins University Press (1996)

4. Haycroft, R., Pronzato, L., Wynn, H., Zhigljavsky, A.: Studying convergence of gradient algorithms via optimal experimental design theory. In: Optimal design and related areas in optimization and statistics, pp. 13 - 37. Springer (2009)

5. Krasnosel'skii, M.A., G.Krein, S.: An iteration process with minimal residues. Mat. Sb. 31(4), 315-334 (1952)

6. Meurant, G.: The block preconditioned conjugate gradient method on vector computers. BIT 24, 623-633 (1984)

7. Pronzato, L., Wynn, H., Zhigljavsky, A.: Asymptotic behaviour of a family of gradient algorithms in $\mathbb{R}^{d}$ and Hilbert spaces. Math. Program. A107(3), 409-438 (2006)

8. Pronzato, L., Wynn, H.P., Zhigljavsky, A.: A dynamical-system analysis of the optimum $s$-gradient algorithm. In: Optimal design and related areas in optimization and statistics, pp. 39-80. Springer (2009)

9. Pronzato, L., Zhigljavsky, A.: Gradient algorithms for quadratic optimization with fast convergence rates. Comput Optim Appl (to appear) (2011). DOI http://dx.doi.org/10.1007/s10589-010-9319-5

10. Saad, Y.: Practical use of polynomial preconditionings for the conjugate gradient method. SIAM J. Sci. Stat. Comp. 6(4), 865-881 (1985)

11. Slater, B.: Gaps and steps for the sequence $n \theta \bmod 1$. Math.Proc.Camb.Phil.Soc. 63, 1115-1123 (1967)

12. van der Vorst, H.A.: Iterative methods for large linear systems. Tech. rep., Math.Inst. Utrecht Univ. (2002) 